\title{
D0-D8-F1 in Massive IIA SUGRA
}

\author{
MARC MASSAR 円 and JAN TROOST P \\ Theoretische Natuurkunde, Vrije Universiteit Brussel \\ Pleinlaan 2, B-1050 Brussel, Belgium
}

\begin{abstract}
We present new supersymmetric solutions of massive IIA supergravity involving D0-branes, a D8-brane and a string. For the bosonic fields we use a general ansatz with $\mathrm{SO}(8)$ symmetry.
\end{abstract}

\section{Introduction}

Since a few years, D-branes [1] are an important ingredient of string theories. They play a crucial role in the more imaginative of recent developments, such as black hole entropy counting, M-theory, Matrix-theory and the ADS/CFT correspondence. Dbranes are defined as spacetime defects on which open strings can end. They can also be found as solutions of the low-energy effective action of the different string theories, i.e. the corresponding supergravity theories.

In [2], it was pointed out that to find the D8-brane as a supergravity solution, you have to turn to Romans' massive IIA supergravity [3] [ [4], in which the mass of the two form in the supergravity multiplet simultaneously acts as a cosmological constant. The mass parameter is proportional to the dual of the ten form field strength associated to the D8-brane.

In general discussions of intersecting brane-solutions in supergravity theories, the special features of massive IIA were mostly ignored. We study some intersections explicitly to see in detail how they arise, and specifically, we will concentrate on configurations involving D0-branes, D8-branes and a string.

In section 2 we discuss briefly the relevant massive IIA SUGRA. In section 3 we give our ansätze and explain our method. In section 4 we discuss solutions.

Recently, a paper which overlaps partly with ours appeared in the hep-th archive [5].

\section{Massive II SUGRA}

The massive IIA $\mathrm{D}=10$ supergravity [0] [3] [4] has the same field content as ordinary IIA supergravity, namely: a metric g, a dilaton $\sigma$, a 2-form $B$, a three-form $C$, a gravitino $\psi$, and a Majorana spinor $\lambda$, but the 2 -form is massive and contains the degrees of freedom of the usual one-form via a generalised Higgs-mechanism. Since we consider purely bosonic backgrounds we do not write the fermion terms explicitly in what follows. As covariant field strengts, we take:

$$
\begin{aligned}
& G=4 d C+6 M(B)^{2} \\
& H=3 d B
\end{aligned}
$$

\footnotetext{
${ }^{1}$ massar@tena4.vub.ac.be

2 troost@tena4.vub.ac.be; Aspirant F.W.O.
} 
where $M$ is the mass parameter. The bosonic part of the lagrangian is

$$
\begin{aligned}
\mathcal{L}= & \sqrt{-g}\left(R-\frac{1}{2}(\partial \sigma)^{2}-\frac{1}{3} e^{-\sigma} H^{2}-\frac{1}{12} e^{\frac{1}{2} \sigma} G^{2}-M^{2} e^{\frac{3}{2} \sigma} B^{2}-\frac{1}{2} M^{2} e^{\frac{5}{2} \sigma}\right) \\
& +\frac{2}{4 ! 4 !}\left(d C d C B+4 M d C B^{3}+\frac{36}{5} M^{2} B^{5}\right) .
\end{aligned}
$$

where the mass of the two-form also appears as a cosmological constant. The procedure to recover ordinary IIA SUGRA (including the one-form) can be found in [4]. The classical equations of motion of the lagrangian can be put in the form:

$$
\begin{aligned}
0= & -R_{m n}+\frac{1}{16} M^{2} e^{\frac{5}{2} \sigma} g_{m n}+\frac{1}{2} \partial_{m} \sigma \partial_{n} \sigma+e^{-\sigma}\left(H_{m}{ }^{r s} H_{r s n}-\frac{1}{12} g_{m n} H^{2}\right) \\
& +2 M^{2} e^{\frac{3}{2} \sigma}\left(B_{m}{ }^{r} B_{n r}-\frac{1}{16} g_{m n} B^{2}\right)+\frac{1}{3} e^{\frac{\sigma}{2}}\left(G_{m}{ }^{r s t} G_{n r s t}-\frac{3}{32} g_{m n} G^{2}\right), \\
0= & -\square \sigma+\frac{5}{4} M^{2} e^{\frac{5}{2} \sigma}+\frac{1}{24} e^{\frac{\sigma}{2}} G^{2}-\frac{1}{3} e^{-\sigma} H^{2}+\frac{3}{2} M^{2} e^{\frac{3}{2} \sigma} B^{2}, \\
0= & D_{r}\left(e^{\frac{\sigma}{2}} G^{r m n p}\right)+\frac{1}{4 ! 3} \epsilon^{\text {mnpqrstuvw }} G_{q r s t} H_{u v w}, \\
0= & D_{p}\left(e^{-\sigma} H^{\text {pmn }}\right)-M^{2} e^{\frac{3}{2} \sigma} B^{m n}-M e^{\frac{\sigma}{2}} G^{m n p q} B_{p q} \\
& +\frac{1}{4 ! 4 !} \epsilon^{\text {mnpqrstuvw }} G_{\text {pqrs }} G_{\text {tuvw }} .
\end{aligned}
$$

To impose supersymmetry on our solutions we set the supersymmetry variations of the fermions to zero - the variations of the bosonic fields automatically vanish. The relevant equations are:

$$
\begin{aligned}
\sqrt{2} \delta \lambda= & -\frac{1}{2} \partial \sigma \cdot \Gamma \epsilon-\frac{5}{8} M e^{\frac{5}{4} \sigma} \epsilon+\frac{3}{8} M e^{\frac{3}{4} \sigma} B \cdot \Gamma^{(2)} \Gamma_{11} \epsilon+\frac{1}{12} e^{-\frac{\sigma}{2}} H \cdot \Gamma^{(3)} \Gamma_{11} \epsilon \\
& -\frac{1}{96} e^{\frac{\sigma}{4}} G \cdot \Gamma^{(4)} \epsilon, \\
\delta \psi_{m}= & D_{m} \epsilon-\frac{1}{32} M e^{\frac{5}{4} \sigma} \Gamma_{m} \epsilon-\frac{1}{32} M e^{\frac{3}{4} \sigma}\left(\Gamma_{m} \Gamma^{(2)} \cdot B+14 \Gamma^{p} B_{p m}\right) \Gamma_{11} \epsilon \\
& +\frac{1}{48} e^{-\frac{\sigma}{2}}\left(\Gamma_{m} \Gamma^{(3)} \cdot H-9 \Gamma^{p q} H_{p q m}\right) \epsilon \\
& +\frac{1}{128} e^{\frac{\sigma}{4}}\left(\Gamma_{m} \Gamma^{(4)} \cdot G+\frac{20}{3} \Gamma^{p q r} H_{p q r m}\right) \epsilon .
\end{aligned}
$$

\section{Ansatz and method}

We concentrate on a supersymmetric configuration involving a D8-brane and a string perpendicular to it, in the z-direction. We impose the following naive projection conditions on the variation parameter of supersymmetry:

$$
\begin{aligned}
\Gamma_{z} \epsilon & =-\Gamma_{012345678} \Gamma_{11} \epsilon=\epsilon \\
\Gamma_{z 0} \Gamma_{11} \epsilon & =\epsilon,
\end{aligned}
$$

These conditions break $1 / 4$ of supersymmetry. Taken together they imply the projection condition for a D0-brane:

$$
\Gamma_{0} \Gamma_{11} \epsilon=\epsilon
$$


such that it is possible to add D0-branes without further breaking supersymmetry. For the bosonic fields we take the most general static ansatz with an $S O(8)$ symmetry and vanishing four-form G:

$$
\begin{aligned}
d s^{2} & =-k(r, z)^{2} d t^{2}+l(r, z)^{2} d z^{2}+m(r, z)^{2}\left(d r^{2}+r^{2} d \Omega_{7}^{2}\right) \\
\sigma & =\sigma(r, z) \\
B & =B_{1} d z \wedge d t+B_{2} d t \wedge d r+B_{3} d z \wedge d r \\
M & =\text { piecewise constant }
\end{aligned}
$$

It contains as special cases the D8-brane (where $k(z)=m(z)$ ), the string solution (where $k(r)=l(r)$ ) and the D0-brane solution (where $l(R)=m(R)$ and $R=$ $\left.\sqrt{r^{2}+z^{2}}\right)$. The Ramond-Ramond two form field strength associated to the D0 brane is contained in the massive B-field, such that our ansatz indeed also applies to the single D0-brane case. Remark that the mass parameter can take different values on either side of a domain wall.

With these ansätze for the projection conditions and bosonic fields, we study the supersymmetry-variations of the fermionic fields. Putting these to zero and studying also the integrablity conditions on the Killing spinor equation, gives conditions on the bosonic fields, enabling us to eliminate all but two of them f . We choose to keep $\sigma$ and $\tilde{m} \equiv e^{-\frac{1}{12} \sigma} m$ :

$$
\begin{aligned}
l & =e^{\frac{1}{12} \sigma} \tilde{m}^{-2} \\
k & =e^{-\frac{7}{12} \sigma} \tilde{m}^{-4} \\
m & =e^{\frac{1}{12} \sigma} \tilde{m} \\
M B_{1} & =-\frac{1}{3} e^{-\frac{4}{3} \sigma} \tilde{m}^{-4}\left(2 \sigma_{z}+15 \frac{\tilde{m}_{z}}{\tilde{m}}\right) \\
M B_{2} & =\frac{2}{3} e^{-\frac{4}{3} \sigma} \tilde{m}^{-4}\left(\sigma_{r}+3 \frac{\tilde{m}_{r}}{\tilde{m}}\right) \\
B_{3} & =0 \\
H_{r z t} & =3 \frac{\tilde{m}_{r}}{\tilde{m}^{7}} .
\end{aligned}
$$

After a lengthy calculation, many seemingly non-trivial equations of motion can then be proven to be linearly dependent. In the end, we only have to solve three coupled differential equations. We can choose them to be:

$$
\begin{aligned}
M & =3\left(\tilde{m}^{2}\right)_{, z} e^{-\frac{4}{3} \sigma} \\
\frac{\delta \mathcal{L}}{\delta \phi} & =0 \\
\frac{\delta \mathcal{L}}{\delta g_{r r}} & =0,
\end{aligned}
$$

The first equation (17) follows from setting the variation of the fermionic fields to zero, while the next two are just two linearly independent equations of motion. The explicit expressions for the equations of motion are:

\footnotetext{
3 The technique described differs slightly from the one used mostly in the literature, where a more restricted ansatz is used, the bosonic equations of motions are then solved and the supersymmetryvariations are checked a posteriori.
} 


$$
\begin{aligned}
\triangle_{r}\left(\tilde{m}^{6}\right)= & -\frac{5}{3} M^{2}\left(\tilde{m} e^{\frac{1}{3} \sigma}\right)^{8}-\frac{4}{3} M\left(\tilde{m} e^{\frac{1}{3} \sigma}\right)^{10} e^{-2 \sigma} \sigma_{, z} \\
\triangle_{r}\left(e^{-2 \sigma}\right)= & \frac{20}{3}\left(\left(\sigma_{, r}\right)^{2} e^{-2 \sigma}\right)-12\left(\frac{\tilde{m}_{, r}}{\tilde{m}}\right)^{2} e^{-2 \sigma}+16 \frac{\tilde{m}_{, r}}{\tilde{m}} \sigma_{, r} e^{-2 \sigma} \\
& +\frac{5}{3} M^{2}\left(\tilde{m} e^{\frac{1}{3} \sigma}\right)^{2}+\frac{26}{3} M\left(\tilde{m} e^{\frac{1}{3} \sigma}\right)^{4} \sigma_{z} e^{-2 \sigma} \\
& +\frac{8}{3}\left(\tilde{m} e^{\frac{1}{3} \sigma}\right)^{6} e^{-4 \sigma}\left(\sigma_{, z}\right)^{2}+2\left(\tilde{m} e^{\frac{1}{3} \sigma}\right)^{6} e^{-4 \sigma} \sigma_{, z z} .
\end{aligned}
$$

Any solution of these coupled differential equations is a solution of massive IIA SUGRA preserving (at least) 1/4 supersymmetry.

\section{Solutions}

Although the differential equations are difficult to solve in general, in specific cases, they simplify drastically. Our analysis covers a lot of cases already discussed in the literature [2] [5] [8] [10], and contains new solutions.

\section{1. $H=0$}

Solving the equation $H=0(\sqrt{16})$, and equation (17) demonstrates that only the flat D8-brane and the isolated D0-brane are solutions. You do not find, for instance, an isolated D0-brane in a D8-brane background, which you might expect to have the required symmetry. But that configuration would be inconsistent, as explained in [9].

\section{2. $B_{2}=0$}

Using the $B_{2}$ equation (14), and the mass equation (17), and then analysing the equations of motion, we find that we are left with the following simple differential equation:

$$
\begin{aligned}
e^{-2 \sigma} & =h(r)+M z \\
\triangle_{r}(h(r)) & =-M^{2} .
\end{aligned}
$$

Adding in the appropriate source term, we find the following solution:

$$
\begin{aligned}
d s^{2}= & -\left(M z+1+\frac{k_{f}}{r^{6}}-\frac{M^{2} r^{2}}{16}\right)^{-\frac{3}{4}}\left(d t^{2}+d z^{2}\right) \\
& +\left(M z+1+\frac{k_{f}}{r^{6}}-\frac{M^{2} r^{2}}{16}\right)^{\frac{1}{4}}\left(d r^{2}+r^{2} d \Omega_{7}^{2}\right) \\
e^{-2 \sigma}= & 1+\frac{k_{f}}{r^{6}}-\frac{M^{2} r^{2}}{16}+M z \\
B= & -\frac{1}{2}\left(M z+1+\frac{k_{f}}{r^{6}}-\frac{M^{2} r^{2}}{16}\right)^{-1} d z \wedge d t
\end{aligned}
$$

Independently, the authors of [5] found this solution in a different approach. It is clear that with the source terms we chose, in the massless case we recover the ordinary string solution [10]. But the interpretation of the solution with the mass term is not clear. Firstly, we do not see an interpretation for the assymptotic geometry. Secondly, 
the metric components of the $\mathrm{z}$ and $\mathrm{t}$ coordinate are equal, leading the authors of (55) to name this solution a massive string solution, but there is no obvious $S O(1,1)$ symmetry, since the metric components depend on the z-coordinate.

\section{3. $B_{1}=0$}

An analogous analysis gives:

$$
\begin{aligned}
\tilde{m} & =n(r) e^{-\frac{2}{15} \sigma} \\
e^{-\frac{8}{5} \sigma} & =2 M z n^{-2} \\
\triangle_{r}\left(n^{5}\right) & =0
\end{aligned}
$$

Taking appropriate source terms, and making a simple $\mathrm{z}^{-}$-coordinate transformation, we find the solution:

$$
\begin{aligned}
d s^{2}= & -(M z)^{\frac{1}{8}}\left(1+\frac{k_{f}}{r^{6}}\right)^{-\frac{13}{8}} d t^{2}+(M z)^{\frac{9}{8}}\left(1+\frac{k_{f}}{r^{6}}\right)^{-\frac{5}{8}} d z^{2} \\
& +(M z)^{\frac{1}{8}}\left(1+\frac{k_{f}}{r^{6}}\right)^{\frac{3}{8}}\left(d r^{2}+r^{2} d \Omega_{7}^{2}\right) \\
e^{\frac{4}{5} \sigma}= & (M z)^{-1}\left(1+\frac{k_{f}}{r^{6}}\right)^{\frac{1}{5}} \\
B= & -\frac{3}{M} \frac{k_{f} r^{5} M z}{\left(k_{f}+r^{6}\right)^{2}} d t \wedge d r \\
= & -\frac{1}{2}\left(1+\frac{k_{f}}{r^{6}}\right)^{-1} d z \wedge d t+\frac{1}{2 M} d\left(\frac{M z}{1+\frac{k_{f}}{r^{6}}} d t\right)
\end{aligned}
$$

The solution assymptotically goes to a flat D8-brane [2]. The other building block in the solution is a harmonic superposition of D0-branes and a string. Note that it does not follow from the ordinary harmonic superposition rules [7]. The two-form B is split in the last line in a part that reminds of the string solution and an exact part that can be interpreted as the D0-brane gauge field $C_{(1)}$.

\section{4. $\quad M=0$}

When $M=0$, we get ordinary IIA SUGRA [3] [4]. Little analysis yields:

$$
\begin{aligned}
\tilde{m} & \equiv n(r)^{1 / 6} \\
\triangle_{r}(h)+n h_{, z z} & =0 \\
\triangle_{r}(n) & =0 \\
C_{(1)} & =h^{-1} d t
\end{aligned}
$$

where $e^{-2 \sigma} \equiv n h^{-\frac{3}{2}}$. This set of differential equations incorporates the flat string solution ( $h=1$ and $\mathrm{n}$ harmonic), the lonely D0-brane solution ( $n=1$ and h harmonic), as well as the harmonic superposition of both $(h=n)$. We want to indicate one more possibility, namely the following solution:

$$
\begin{aligned}
d s^{2}= & -(a|z|)^{-\frac{7}{8}}\left(1+\frac{k_{f}}{r^{6}}\right)^{-\frac{3}{4}} d t^{2}+(a|z|)^{\frac{1}{8}}\left(1+\frac{k_{f}}{r^{6}}\right)^{-\frac{3}{4}} d z^{2} \\
& +(a|z|)^{\frac{1}{8}}\left(1+\frac{k_{f}}{r^{6}}\right)^{\frac{1}{4}}\left(d r^{2}+r^{2} d \Omega_{7}^{2}\right)
\end{aligned}
$$




$$
\begin{aligned}
e^{-2 \sigma} & =\left(1+\frac{k_{f}}{r^{6}}\right)(a|z|)^{-\frac{3}{2}} \\
M & =0 \\
B & =-\frac{1}{2}\left(1+\frac{k_{f}}{r^{6}}\right)^{-1} d z \wedge d t \\
C_{(1)} & =\frac{1}{a|z|} d z
\end{aligned}
$$

This solution consists of an eight-dimensional wall of D0-branes and a string perpendicular to it. The behavior of the potential is similar to that of a point charge in one dimension.

\section{Conclusions}

We considered the problem of finding new supersymmetric SUGRA solutions preserving $1 / 4$ supersymmetry. Starting from a quite general ansatz, corresponding to a superposition of D0-branes, a string and a D8-brane with $S O(8)$ symmetry, we studied the supersymmetry variations of the fermionic fields and thus simplified the equations of motion. We cover all cases of combinations of these branes in the literature. Moreover, we discovered some new solutions. One of them reduces in the massless case to the ordinary fundamental string solution, while the interpretation in the massive case is unclear. This solution was independently found in [5]. Another solution consists of a harmonic superposition of D0-branes and a string in a D8-brane background. Our system of differential equations may well contain other non-trivial configurations with interesting physical interpretations. For instance the phenomenon of the creation of a string in the D0-D8 brane system [11] could be approached with our ansatz. Moreover it would be interesting to apply the masssive T-duality rules [2] [12 to our new solutions to gain more insight in their interpretation. We also plan to study other supergravity configurations with our approach.

Acknowledgments: We would like to thank Marco Billo, Ben Craps, Frederik Denef, Frederik Roose, Rodolfo Russo and Walter Troost for discussions. We gladly made use of the GRTensorM 1.2 Mathematica package [13]. This work was supported in part by the European Commission TMR programme ERBFMRX-CT96-0045 in which the authors are associated to K.U. Leuven. 


\section{References}

1. J. Polchinski, Phys. Rev. Lett. 75, 4724 (1995) hep-th/9510017; J. Polchinski, hep-th/9611050

2. E. Bergshoeff, M. de Roo, M. B. Green, G. Papadopoulos and P.K. Townsend, Nucl. Phys B470 (1996) 113, hep-th/9601150.

3. F. Giani and M. Pernici, Phys. Rev D30 (1984) 325

4. L. J. Romans, Phys. Lett. B169 (1986) 374.

5. B. Janssen, P. Meessen and T. Ortin, hep-th/9901078

6. H. Lü, C. N. Pope and J. Rahmfeld, hep-th/9805151

7. G. Papadopoulos and P. Townsend, Phys. Lett. B380 (1996) 273, hepth/9603087; A. Tseytlin, Nucl. Phys. B475 (1996) 179, hep-th/9604035; J. Gauntlett, D. Kastor and J. Traschen, Nucl. Phys B478 (1996) 544, hepth/9604179

8. M.J. Duff and J. X. Lu, Nucl. Phys. B411 (1994) 473, hep-th/9306052 and references therein.

9. J. Polchinski and A. Strominger, Phys. Lett. 388 (1996) 736 hep-th/9510227; A. Strominger, Phys. Lett. B383 (1996) 44, hep-th/9512059

10. A. Dabholkar, G. W. Gibbons, J. A. Harvey and F. Ruiz-Ruiz, Nucl. Phys. B340 (1990) 33.

11. C. Bachas, M.Douglas, M. Green, JHEP 9707(1997) 002, hep-th/9705074;PeiMing Ho and Yong-Shi Wu,Phys.Lett. B420 (1998) 43, hep-th/9708137; PeiMing Ho, Miao Li and Yong-Shi Wu, Nucl.Phys. B525 (1998) 146, hepth/9706073; U.Danielsson, G Ferreti, I. Klebanov, Phys. Rev. Lett. 79 (1997) 1984, hep-th/9705084; O. Bergman, M. Gaberdiel, G. Lifschytz, Nucl. Phys. B 509 (1998) 194, hep-th/9705130; M. Billo, P. Di Vecchia, M. Frau, A. Lerda, I. Pesando, R. Russo, S. Sciuto, Nucl.Phys. B526 (1998) 199, hep-th/9802088

12. E. Bergshoef, C. Hull and T. Ortín, Nucl. Phys B451 (1995) 547, hep-th/9504081

13. P. Musgrave, D. Pollney and K. Lake, GRTensor II, http://www.astro.queensu.ca / grtensor/ 